\title{
La formación docente inicial del profesor de Historia en Brasil: temas, reflexiones y desafíos*
}

\author{
Erinaldo Cavalcanti \\ Universidad Federal de Sul y Sudeste de Pará (Unifesspa), Brasil \\ ericontadordehistorias@gmail.com \\ https://orcid.org/0000-0002-9912-5713
}

\section{RESUMEN}

Existe un consenso relativo en la bibliografía especializada sobre el hecho de que el libro de texto sigue siendo una poderosa herramienta de trabajo de los docentes de la educación primaria y secundaria en Brasil. Debido a las dimensiones geográficas de Brasil, este artículo hace un recorte y analiza los diseños curriculares de las carreras de grado en Historia ofrecidos en universidades federales en la región amazónica de Brasil para comprender cómo los libros de texto son analizados durante el período de formación inicial de los docentes. Como opción metodológica, se procedió al análisis cuantitativo y cualitativo de las matrices curriculares de los cursos ofrecidos en las universidades federales de la región amazónica de Brasil. Se analizó el en estas universidades y, de manera específica, el papel que juega el debate sobre el libro de texto de historia en las referidas carreras de grado. Para ello, fueron analizadas las asignaturas obligatorias dedicadas al estudio de la enseñanza de historia: los libros de texto, sus objetivos y sus cargas horarias. El análisis muestra que, en los grados estudiados, la división clásica de temas prevalece en el modelo eurocéntrico cuadripartito y que no hay consenso sobre el lugar que ocupa el debate sobre la enseñanza de la historia. Además, se identifica una ausencia casi completa de estudios sobre libros de texto.

Palabras clave: enseñanza de la historia; libro de texto; formación del profesorado; Brasil.

Cómo citar: Cavalcanti, E. (2020). La formación docente inicial del profesor de Historia en Brasil: temas, reflexiones y desafíos. Ciencias Sociales y Educación, 9(18), 113-135. https://doi.org/10.22395/csye.v9n18a5 Recibido: 4 de agosto de 2020

Aprobado: 20 de agosto de 2020. 


\section{The Initial formation of the History Teacher in Brazil: Topics, Reflections and Challenges}

\section{ABSTRACT}

There is a relative consensus regarding the specialized bibliography on the fact that the book is still a tremendous and powerful work tool for teacher in the elementary and high school in Brazil. Due to the geographical dimension of Brazil, this article concentrates its research interests in the federal universities in the Amazonian region of Brazil in order to understand how the text books are analyzed during the initial formation process of teachers. As a methodological option, this research employed a qualitative and quantitative analysis of the curricular arrays of the courses offered in the federal universities of the Amazonian region of Brazil. We analyzed the place occupied by the tea- ching of history in these universities, and, specifically, the role played by the debate on the history text books in the said programs. For that, the research analyzed mandatory courses dedicated to the study of the history teaching: the texts books, their objective and their workload. The analysis showed that, in the studies degrees, the classical topic division still prevails under a quadripartite Eurocentric model and that there is no consensus on the place occupied by the debate on the teaching if history. Furthermore, the research identifies an almost complete absence of studies on text books.

Keywords: history teaching; text book; teacher's education; Brazil.

\section{A formação docente inicial do professor de História no Brasil: temas, reflexões e desafios}

\section{RESUMO}

Há um consenso relativo na bibliografia especializada sobre o fato de o livro didático continuar sendo uma poderosa ferramenta de trabalho para professores do ensino fundamental e médio no Brasil. Devido às dimensões geográficas do Brasil, este artigo recorta e analisa os desenhos curriculares dos programas de graduação em História oferecidos nas universidades federais da região amazônica do Brasil para entender como os livros didáticos são analisados durante o período de formação inicial de professores. Como opção metodológica, foi realizada uma análise quantitativa e qualitativa das matrizes curriculares dos cursos oferecidos nas universidades federais da região amazônica do Brasil. Foi analisado o lugar que o ensino de história ocupa nessas universidades e, especificamente, o papel que o debate sobre o livro didático de história desempenha nos referidos cursos de graduação. Para tanto, foram analisadas as disciplinas obrigatórias dedicadas ao estudo do ensino de história: os livros didáticos, seus objetivos e sua carga horária. A análise mostra que, nos níveis que foram estudados, prevalece a clássica divisão de temas no modelo eurocêntrico e que não há consenso sobre o lugar ocupado pelo debate sobre o ensino de história. Além disso, há uma ausência quase total de estudos sobre livros didáticos.

Palavras-chave: Brasil; ensino de história; formação de professores; livro didático. 


\section{Introducción}

En primer lugar, es importante aclarar a los lectores no nativos algunas denominaciones sobre la formación inicial de docentes en Brasil. Esta formación consiste en una carrera de grado - ofrecida por universidades públicas y privadas y por algunos institutos de educación técnica y superior-con duración mínima de cuatro años. Para que se pueda desempeñar la docencia en los niveles primario y secundario (enseñanza fundamental y enseñanza media), es obligatorio realizar la carrera de formación superior. En Brasil, el nivel superior corresponde a la formación universitaria.

Para ejercer la actividad docente, el profesional necesita un título de grado en una carrera de licenciatura. En otras palabras, ese grado académico habilita a los profesionales a ejercer la enseñanza en los niveles primario o secundario. Las carreras de licenciatura de diversas áreas del conocimiento ofrecen en sus planes de estudio un conjunto de asignaturas, llamadas pedagógicas, para preparar a los profesionales al ejercicio de la enseñanza. El Ministerio de Educación regula un conjunto de normativas mediante las cuales exige una cantidad mínima de horas para las asignaturas pedagógicas. Actualmente, la resolución vigente (Resolución 2, 2019) determina cuatrocientas horas para las asignaturas o actividades comúnmente llamadas "prácticas de enseñanza", y otras cuatrocientas horas para las prácticas docentes.

Se hace necesaria una breve explicación para ubicar el presente artículo. Las reflexiones presentadas aquí son resultado del proyecto Historia de la enseñanza, libros de texto y formación del profesorado: entre prácticas y representaciones, cuyo objetivo general es analizar los diseños curriculares de las carreras de licenciatura en Historia que se ofrecen en las universidades federales de Brasil para comprender cómo los debates sobre la enseñanza de la historia y los libros de texto se problematizan durante la formación inicial de los docentes. Este proyecto está vinculado a la línea de investigación Enseñanza de Historia, Narrativas y Documentos del programa de posgrado en Historia de Unifesspa y al laboratorio y grupo de investigación iTemnpo ${ }^{1}$.

El presente artículo se inserta en ese espacio de reflexión y propone un análisis sobre algunos temas que involucran la historia y su enseñanza, entendida

Como resultado de la investigación llevada a cabo en iTemnpo sobre el libro de texto de historia, a fines de 2019, estas investigaciones se convirtieron formalmente en parte del proyecto de investigación Las ciencias sociales en los iberoamericanos: análisis de libros de texto. Es un proyecto de investigación dirigido por la Universidad de Zaragoza (España) a través del Centro Internacional de la Cultura Escolar Berlonga de Duero-Soria de la Universidad de Zaragoza y actualmente cuenta con ocho países: España, Portugal, Chile, Argentina, Brasil, Colombia, México y Ecuador y con más de veinte universidades participantes. El objetivo general del proyecto es comprender cómo se tratan los eventos/tiempos considerados esenciales en la historia de Iberoamérica (descubrimiento, conquista, colonización, independencia y actualidad) en libros de texto en español, portugués e iberoamericano de diferentes épocas históricas. 
como un campo de disputas y como un lugar de conocimiento (poder) y formación docente. Se presentan algunas reflexiones sobre ciertos temas que se encuentran en las asignaturas/materias de la licenciatura en Enseñanza de la Historia, ofrecidas por universidades federales ubicadas en la región amazónica de Brasil. De manera específica, se reflexiona acerca de cómo aparecen las discusiones sobre la enseñanza de historia y el libro de texto en las carreras analizadas.

Se utilizan los diseños curriculares de los cursos introductorios de grado, también llamados Proyecto Político Pedagógico (PPP) o Proyecto Político Curricular (PPC), como una opción para problematizar algunos objetos de estudio como la enseñanza y el libro de texto en las referidas carreras de licenciatura. El análisis demuestra que los diseños curriculares investigados han tematizado predominantemente la historia europea y mantienen la clásica periodización cuadripartita: historia antigua, medieval, moderna y contemporánea. Además, existe poco interés en reflexionar sobre la enseñanza de la historia y menos sobre el libro de texto.

Es conveniente ubicar la Amazonía brasileña. El mapa siguiente (figura 1) muestra la localización de área en que se encuentra las instituciones investigadas.

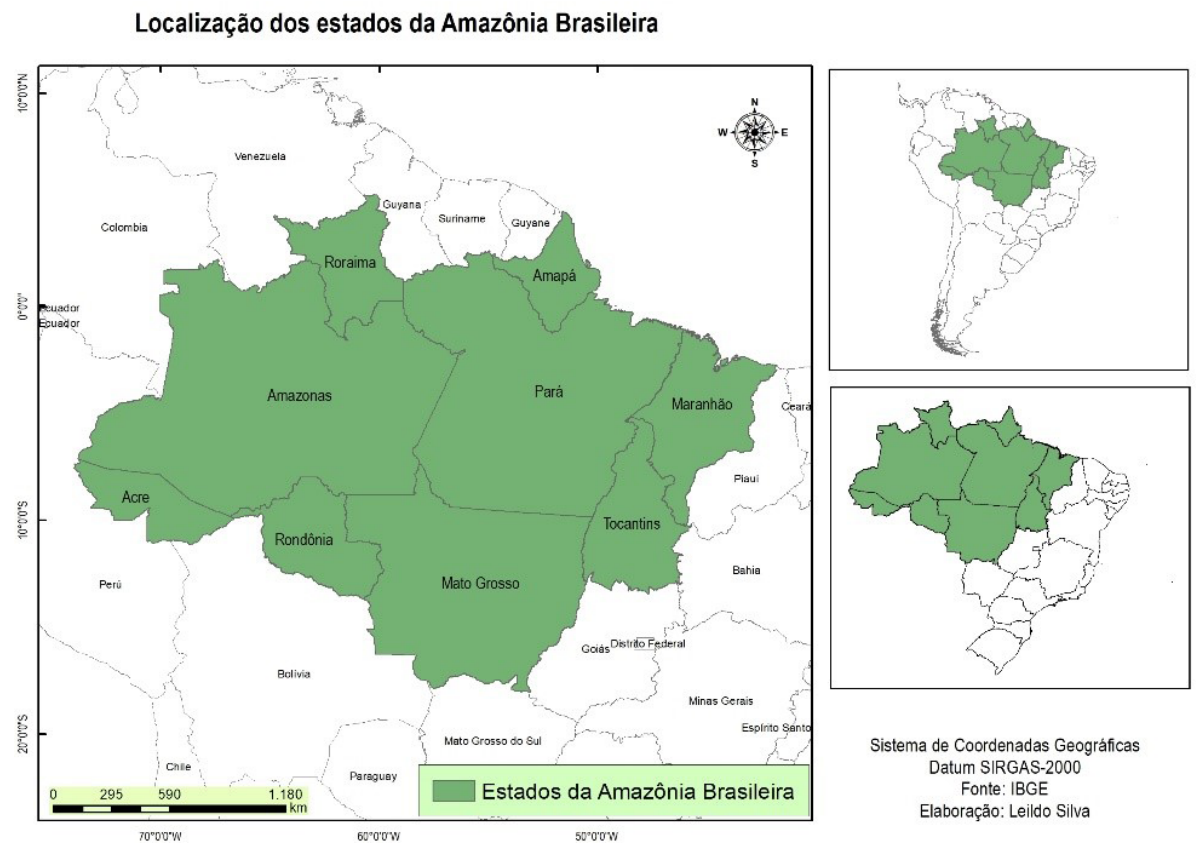

Figura 1. Localización de la Amazonia brasileña

Fuente: elaboración propia. 
Según el Instituto Brasileño de Geografía y Estadística (IBGE), la Amazonía brasileña "tiene un área aproximada de $5.217 .423 \mathrm{~km}^{2}$, que corresponde aproximadamente al $61 \%$ del territorio brasileño" (IBGE, 2020). Las instituciones investigadas en el presente artículo san las siguientes: Universidad Federal de Amazonas (UFAM), Universidad Federal de Acre (UFAC), Universidad Federal de Amapá (Unifap), Universidad Federal de Pará (UFPA), Universidad Federal del Sur y Sudeste del Pará (Unifesspa), Universidad Federal del Oeste del Pará (Ufopa), Universidad Federal de Rondônia (UNIR), Universidad Federal de Roraima (UFRR), Universidad Federal de Tocantins (UFT), Universidad Federal de Mato Grosso (UFMT) y Universidad Federal de Maranhão (UFMA).

¿Qué se enseña en las carreras de licenciatura en Historia de esas instituciones? ¿Significa esta pregunta reflexionar sobre qué temas deben ser estudiados por los estudiantes en el período de formación inicial del profesor de Historia? ¿Qué pasados deben continuar formalmente, a través de la enseñanza de la historia, para tejer las narraciones sobre las historias de los hombres en el tiempo? ¿Qué pasado debe estar presente en nuestra vida diaria en el salón de clases? ¿Qué presentes deben configurarse a través de las lecturas de los informes pasados en los libros de texto? ¿Qué futuros se deben proyectar por medio de las narrativas presentes en los libros de texto que se utilizan en las clases de historia? ¿Cómo deben utilizarse estos libros de texto en el ejercicio de los profesionales de la enseñanza que trabajan en la educación primaria y secundaria? ¿Cómo se analizan estos materiales durante la formación de esos profesionales?

Seguramente no hay respuestas listas y terminadas a ese conjunto de preguntas. Diferentes autores han contribuido a solucionar algunos de esos problemas y nos ayudan con el ejercicio de la reflexión. Algunos se utilizan como referencia en este texto y contribuyen a los análisis propuestos.

La Historia —como área de conocimiento y espacio para la formación del profesorado a nivel de grado en universidades de Brasil- ha construido diferentes "propuestas" de formación de docentes a través de sus diseños curriculares. La configuración de esas propuestas prioriza ciertos temas y excluye o minimiza las reflexiones sobre la enseñanza y el libro de texto durante la formación inicial de los profesores. Para apoyar esta lectura, los planes curriculares de las carreras de Historia pueden ser apropiados como documentos que indican cómo las asignaturas obligatorias se distribuyen por temas de estudio. De esta manera, estas disciplinas garantizan el debate, la reflexión y el aprendizaje de ciertos temas a la vez que imposibilitan otros debates, reflexiones y aprendizajes durante la formación inicial del profesorado. 


\section{El objeto de estudio: una mirada a partir de la bibliografía especializada}

Es importante registrar que la formación docente es ampliamente tematizada por diferentes investigadores y por distintos objetos de investigación, lo que no significa que ese sea un tema agotado. Las reflexiones de la bibliografía especializada ofrecen valiosas contribuciones para mostrar la pluralidad de abordajes y la importancia que la temática merece, en especial en el momento actual en que la educación y la formación docente en Brasil sufren ataques de forma sistemática.

En esta perspectiva, las reflexiones de Maurice Tardif (2004) son importantes en tanto postulan que los conocimientos y las prácticas docentes son múltiples y heterogéneas. Por lo tanto, no existe relación de determinismo entre los documentos prescritos y las acciones de los maestros en el ejercicio de la enseñanza. En sintonía con lo anterior, las contribuciones de Ana Maria Monteiro (2007) también son valiosas para argumentar que existe una autonomía relativa en las actividades desarrolladas por los profesores en el ejercicio de su profesión. Una autonomía construida a partir de la trayectoria personal y profesional, de las referencias sociales y culturales compartidas por los profesores.

Los desafíos en la formación de los docentes van más allá de las barreras políticas del tiempo y del espacio; por supuesto, en cada momento y en cada lugar las disputas adquieren configuraciones específicas. Al analizar la formación de los docentes en Latinoamérica, la profesora Margarita Victoria Rodríguez destaca el papel del estado como agente promotor de los principales cambios implementados desde la década de 1990 en el proceso de globalización. Rodríguez señala las reformas implementadas en Chile, Argentina y Uruguay; muestra los desafíos y disputas sobre el proceso de capacitación del profesorado y apunta que todavía prevalece una distancia entre instituciones de educación superior, donde se forman los docentes, y la propia educación primaria y secundaria. En sus palabras "hay una gran distancia entre los formadores de los profesores y la realidad escolar" (Rodríguez, 2008, p. 19).

Las reflexiones tienen lugar en diferentes países que eligen una amplia gama de análisis. Desde esa perspectiva, las reflexiones de la profesora María Paula González (2004-2005) son importantes a la hora de analizar las relaciones entre las prácticas docentes, la transmisión de la historia reciente y el currículo en Argentina. Para la autora, los profesores movilizan un conjunto variado de factores en el ejercicio de la enseñanza y, por lo tanto, deben entenderse "como actores claves y a sus lecturas e interpretaciones del currículum como actos de resignificación activa" (González, 2004-2005, p. 2). 
La profesora Maria do Céu de Melo (2015), de la Universidad del Miño, hace un análisis de la formación del profesor de Historia en Portugal, en el que señala que la estructura y organización de los cursos universitarios en la Unión Europea se configuran a través del proyecto ganador que está diseñado en el Tratado de Bolonia, el cual fue aprobado en 2001 e implementado en diferentes ritmos y formas. Al analizar el máster profesional en Enseñanza de Historia, ofrecido en esa universidad, Melo destacó que es un requisito obligatorio que el estudiante tenga formación en el primer ciclo de Historia. A continuación, muestra cómo se configura la estructura curricular de ese período de formación inicial:

Las unidades curriculares (UCs) (disciplinas) de este ciclo de estudios se centran en los conocimientos básicos y las competencias de la historia, articulando de manera multidisciplinaria tiempos y espacios históricamente diversos: prehistoria, civilizaciones antiguas, civilización griega y romana, arte clásico, Edad Media (Europa y Portugal), descubrimientos y expansión portuguesa, arte medieval, Edad Moderna (Europa y Portugal), arte moderno, Edad Contemporánea (Mundo y Portugal), arte contemporáneo, y temas de otras ciencias sociales como, sociología o antropología (opcional). (Melo, 2015, p. 42)

A su vez, el profesor Pagès (2004) señala, al analizar el proceso de formación docente del profesor de Historia de la escuela secundaria en España, que el perfil de ese profesional es una construcción histórica con variados e importantes elementos del campo de conocimiento epistemológico e historiográfico. Todavía cuestiona si ese conocimiento es suficiente para enseñar Historia en la escuela secundaria -y quizá en toda la educación primaria y secundaria para el caso de Brasil—, ya que la formación de los docentes ha priorizado la adquisición de conocimientos específicos sobre los contenidos que se van a enseñar. Para Pagès (2004), enseñar es un acto de comunicación. Por lo tanto, es necesario que los que enseñan tengan formación para "esta comunicación", conozcan los medios, los discursos, los instrumentos y el contexto en el que se construye la comunicación, es decir, la enseñanza. Del mismo modo, es necesario que el comunicador conozca al público a que se dirige su comunicación, es decir, el docente de Historia debe tener conocimiento sobre los estudiantes a los que se propone enseñar. En este sentido, Pagès (2004) se pregunta por

lo que se comunica, este caso el conocimiento histórico, y cómo debemos comunicarlo para obtener aprendizajes. Y, además, el contexto en el que se realiza la comunicación, contexto que incluye desde las finalidades o propósitos educativos que la administración educativa otorga a los saberes escolares, el contexto social y cultural, hasta la institución en la que se realiza y la organización espacio-temporal en la que tiene lugar (organización del espacio aula y tiempo de duración de la comunicación). (p. 157)

Los debates sobre la educación de los profesores implican directamente la discusión sobre los diseños curriculares. No es el objetivo de este artículo 
presentar una revisión bibliográfica del currículo, ya que ello requeriría otro ensayo para las reflexiones necesarias. Sabemos que el currículo es un tenso campo de combate. Es un espacio de luchas y enfrentamientos. Es un territorio de disputas, en la interpretación de Arroyo (2013). Es el lugar de las batallas de proyectos políticos y sociales, como defienden Moreira y Silva (2000); un espacio de invención, creación y representación del mundo (Monteiro, 2013). En este sentido, el currículo no es solo un lugar de disputas sobre la selección de contenidos y metodologías de enseñanza, sino que "el plan de estudios es una opción cultural, un proyecto cultural selectivo, una selección cultural de ciertos grupos" (Guimarães y Couto, 2008, p. 118).

La enseñanza de la historia como objeto de preocupación y análisis de la ciencia histórica se entrelaza con las relaciones tejidas en el tiempo. Los usos de la historia, a través de su enseñanza, están estrechamente relacionados con las diferentes lecturas interpretativas que los hombres elaboran sobre su tiempo. Estas lecturas ofrecen, por lo tanto, una orientación sobre las diferentes formas de actuar en el espacio político. Así, se decide qué enseñar, cómo enseñar y a quién enseñar. Tales decisiones se fabrican en las relaciones de poder que los hombres temen en y con el tiempo.

$\mathrm{Al}$ analizar algunos desafíos del proceso de enseñanza y aprendizaje de la historia en la formación inicial del profesorado, Cristiani Bereta da Silva y Luciana Rossato destacan el crecimiento y la importancia que han brindado las reflexiones sobre la educación histórica. Para las autoras, el crecimiento y el contenido de la investigación nos permiten afirmar "que progresaremos poco en la enseñanza de la historia escolar y en la capacitación de maestros para la educación primaria y secundaria si no conseguimos comprender los procesos cognitivos y de aprendizaje involucrados en la elaboración de ideas históricas" (Silva y Rossato, 2013, p. 82).

La enseñanza de historia y los libros de texto son también objetos de las más variadas reflexiones; consisten en espacios de distintas y tensas disputas o de guerras de narrativas (Laville, 1999). Esas disputas políticas pueden implicar el acceso o no a una narrativa socialmente reconocida sobre la historia de otros pueblos, espacios y tiempos. En estos términos, el libro de texto y la enseñanza de historia son espacios en disputa para la historia, como señalan las investigaciones de Abud (1998), Bittencourt (2009), Cassiano (2017), Cavalcanti (2016, 2018a, 2018b), Rocha, Reznik y De Souza (2017), Oliveira, Cainelli y Oliveira (2008), Rocha (2019), Miranda y Luca (2004), Munakata (2012) y Choppin (2004).

Las disputas sobre qué pasados se deben estudiar y cómo estudiarlos no solo ocurren en el campo estrictamente científico de la historia. Se intensificaron - y todavía persisten- cuando la enseñanza de la historia se convirtió en un 
instrumento formal de la educación como política estatal. No solo en Brasil, la enseñanza de la historia fue y sigue siendo, en diferentes momentos, un campo de luchas y debates entre plataformas políticas que cuestionan el control o la conducción de los proyectos de las sociedades (Mathias, 2011). Los enfrentamientos que ocurrieron sobre la retirada de la enseñanza obligatoria de historia en la escuela secundaria son bastante significativos. Estos enfrentamientos aún se desarrollan intensamente en torno a la Base Nacional de Currículos Comunes (BNCC). ${ }^{2}$

\section{Procedimientos metodológicos}

Para proceder metodológicamente, se optó inicialmente por un enfoque cuantitativo de los PPC. Primero se identificaron cuántas instituciones ofrecen el curso de Historia en los campus de su sede. Ese enfoque considera once universidades. A continuación, se realizó un mapeo de cuántas asignaturas obligatorias se ofrece en cada PPC y cuáles son los temas estudiados. Posteriormente, se comenzaron a registrar cuántas asignaturas obligatorias están destinadas a los debates sobre la enseñanza de la historia y cuántas se dirigen a las reflexiones sobre los libros de texto.

A través de la herramienta de identificación de palabras, se buscaron las siguientes palabras en los documentos de los once diseños curriculares: "enseñanza de la historia", "libro de texto", "material didáctico" y "manual didáctico". Este procedimiento permitió identificar cuántas asignaturas ofrecen reflexiones sobre la enseñanza de la historia y el libro de texto durante el período de formación inicial de los profesores. Así, fue posible analizar el lugar que esas asignaturas ocupan en los diseños curriculares, su carga, su programa de contenidos y sus objetivos.

Es importante resaltar que esa opción metodológica no se limita al conteo numérico de las asignaturas que se ocupan de enseñanza y libros de texto. Fundamentalmente, el interés es comprender qué asignaturas y temas se ofrecen, es decir, cuáles son los contenidos estudiados en cada una de ellas. Esos datos permiten comprender y analizar la división y distribución del tiempo entre los componentes curriculares. La división ocurre de manera tensa, a través de disputas, negociaciones y confrontaciones. Por lo tanto, la división y destrucción de temas por asignaturas no son naturales ni se limitan a una cuestión matemática. Al contrario, son, ante todo, una cuestión política entretejida en las relaciones de poder fabricadas en ese momento.

La Base Nacional de Currículos Común fue aprobada en 2018 y estableció un conjunto variable de cambios en la educación primaria y secundaria. Entre estos cambios, la oferta de enseñanza de Historia ya no es obligatoria en la escuela secundaria. Sobre el debate sobre el proceso de discusión de BNCC véase Miranda (2019). 
Se accedió a los documentos de esos diseños curriculares y se recopilaron durante la primera etapa del proyecto, que fue iniciado en el segundo semestre de 2016 (a excepción de la Ufopa, cuyo curso fue creado en el segundo semestre de 2018). Por lo tanto, es posible que algunos diseños hayan sufrido cambios en ese intervalo de tiempo. Los diseños curriculares analizados aquí se encuentran en las sedes de las respectivas instituciones, a excepción de la Universidad Federal de Tocantins (UFT), cuyo diseño es del curso ofrecido en la ciudad Porto Nacional.

Se implementó un segundo procedimiento metodológico para proceder con el análisis cualitativo de los datos catalogados. Este análisis se basa en la lectura e interpretación de los enunciados presentes en los planes de curso de cada asignatura analizada. Así, después de identificar cuáles y cuántas asignaturas se dirigen a estudios sobre la enseñanza de la historia y los libros de texto, se comenzó a analizar lo que esas asignaturas curriculares seleccionaron como temas de estudio específicos, es decir, lo que los diseños curriculares definieron, en un amplio universo de debates relacionados con la enseñanza de la historia y los libros de texto, como necesarios para enseñar y aprender durante la formación inicial del docente de Historia. Esa metodología de reflexión está presente en todo el trabajo aquí presentado.

Esta investigación ha permitido construir un amplio panorama e identificar el lugar que ocupa la enseñanza de la historia en los diseños curriculares de los cursos analizados. También es posible observar cómo esos cursos dividen los temas trabajados y seleccionan la secuencia para cada contenido. Además, demuestra lo que se considera obligatorio y opcional en términos de estudios para la formación de los futuros docentes de la educación primaria y secundaria.

\section{Análisis de los datos de la investigación}

Se entiende que no hay elemento que condicione una relación mecánica entre lo que está escrito y prescrito en los diseños curriculares y su aplicabilidad. No existe una fuerza que establezca una factibilidad entre los enunciados de los programas de contenidos y su ejecución por parte del docente responsable por su enseñanza. Entre lo prescrito y lo experimentado hay un universo de tensiones, negociaciones y acuerdos que promueven otras configuraciones para las materias. Hay muchas prácticas desarrolladas que no se describen en los documentos. Hay muchos temas que actúan en esta etapa cuya ejecución no sigue la determinación irrestricta del currículum prescriptivo oficial. No debemos olvidar que la implementación —o desarrollo- de las materias, como regla, sigue la dinámica propia de los programas de curso elaborados por los docentes responsables. Por lo tanto, esos diseños deben ser considerados vestigios, la 
evidencia de un universo tenso, denso y complejo de problemas en disputas y metamorfosis permanentes.

Sin embargo, eso no significa que tengamos que disminuir la importancia de los diseños curriculares y los discursos que se registran en sus comentarios. No. Los diseños demuestran y dan una interpretación sobre la comprensión de la historia como ciencia, como un lugar de formación docente. Además, nos permiten entender cómo los docentes que actuaron en la construcción de cada diseño entendieron, y entienden, lo que debe ser importante para los profesionales que se convierten en profesores de Historia.

Como cualquier otro documento, los diseños curriculares necesitan ser analizados cuidadosamente, pues constituyen una especie de ADN de las carreras y de los departamentos de Historia en las universidades de Brasil. Esos diseños representan un conjunto de discursos y prácticas que caracterizan la forma de hacer de la historia una ciencia en nuestras universidades; ofrecen y dan a leer la configuración que adquirieron las carreras de formación de profesores de Historia; demuestran la concepción de la historia a través de sus enunciados, sus materias y comentarios; expresan sus afiliaciones teóricas y conceptuales; señalan enfoques y distancias con diferentes significados de la historia; indican lo que consideran importante en la formación de graduados de Historia; informan qué preguntas son obligatorias para el profesional del área y qué preguntas son opcionales; presentan los diferentes significados de las categorías analíticas que componen esta ciencia, como "narrativa", "documento", "hecho/evento", "tiempo" (por nombrar solo algunos).

La configuración temática de los diseños analizados se estructura a través de la división clásica de la historia en cuatro períodos. La tabla 1 presenta una visión general sobre la carga horaria y la distribución del número de temas relacionados con las llamadas historia antigua, historia medieval, historia moderna e historia contemporánea en los cursos analizados.

Tabla 1. Relación institución/carga-horaria del curso y materias (asignaturas)

\begin{tabular}{lcccccc}
\hline \multirow{2}{*}{$\begin{array}{c}\text { Institución } \\
\text { (año de aprobación }\end{array}$} & $\begin{array}{c}\text { Carga } \\
\text { horaria } \\
\text { del PPC) }\end{array}$ & \multicolumn{5}{c}{ Cantidad de asignaturas obligatorias } \\
\cline { 3 - 7 } & $\begin{array}{c}\text { total } \\
\text { (horas) }\end{array}$ & Total & $\begin{array}{c}\text { Historia } \\
\text { antigua }\end{array}$ & $\begin{array}{c}\text { Historia } \\
\text { medieval }\end{array}$ & $\begin{array}{c}\text { Historia } \\
\text { moderna }\end{array}$ & $\begin{array}{c}\text { Historia } \\
\text { contemporánea }\end{array}$ \\
\hline UFAM (2006) & 2.865 & 41 & 1 & 2 & 1 & 2 \\
\hline Unifap (2007) & 3.420 & 44 & 2 & 1 & 2 & 2 \\
\hline UFMT (2009) & 3.268 & 39 & 2 & 2 & 2 & 2 \\
\hline UFPA (2011) & 3.260 & 45 & 1 & 1 & 1 & 3 \\
\hline UFT (2011) & 2.805 & 36 & 1 & 1 & 1 & 2 \\
\hline
\end{tabular}




\begin{tabular}{lcccccc}
\hline \multirow{2}{*}{$\begin{array}{c}\text { Institución } \\
\text { (año de aprobación }\end{array}$} & $\begin{array}{c}\text { Carga } \\
\text { horaria } \\
\text { del PPC) }\end{array}$ & \multicolumn{5}{c}{ Cantidad de asignaturas obligatorias } \\
\cline { 5 - 8 } & thoras) & Total & $\begin{array}{c}\text { Historia } \\
\text { antigua }\end{array}$ & $\begin{array}{c}\text { Historia } \\
\text { medieval }\end{array}$ & $\begin{array}{c}\text { Historia } \\
\text { moderna }\end{array}$ & $\begin{array}{c}\text { Historia } \\
\text { contemporánea }\end{array}$ \\
\hline UFRR (2012) & 2.810 & 38 & 1 & 1 & 1 & 2 \\
\hline UFAC (2013) & 2.930 & 39 & 1 & 1 & 1 & 2 \\
\hline UFMA (2014) & 2.865 & 36 & 1 & 1 & 2 & 2 \\
\hline UNIR (2014) & 3.560 & 32 & 1 & 1 & 1 & 2 \\
\hline Unifesspa (2018) & 3.216 & 45 & 1 & 1 & 2 & 2 \\
\hline Ufopa (2018) & 3.470 & 37 & 1 & 1 & 1 & 2 \\
\hline Total & 34.469 & 432 & 13 & 13 & 15 & 23 \\
\hline
\end{tabular}

Fuente: elaboración propia.

Este muestreo es una descripción general. Hay otras asignaturas distribuidas en los diseños curriculares sobre historia de América, teoría y metodología de la historia e historia de Brasil. Sin embargo, desde el punto de vista de la distribución temporal de los contenidos trabajados en los cursos analizados, se puede ver una división secuencial y cronológica de las disciplinas ofrecidas en cada período. En otras palabras, los primeros temas curriculares son aquellos que tratan de un marco de tiempo más distante (o más antiguo) y los últimos componentes abordan temas más cercanos a los tiempos actuales.

En todos los cursos analizados, se ofrece al menos una asignatura que abarca la llamada historia antigua, con algunas variaciones en los títulos y en los objetivos a los que están destinadas. En todos los diseños curriculares, esa es una asignatura obligatoria ofrecida en el primer semestre de los cursos.

A continuación, la secuencia de los temas de estudio obligatorios de las carreras muestra las asignaturas que analizan la llamada Edad Media. En otras palabras, desde el segundo o tercer período, los cursos de grado abordan las relaciones que construyen el universo medieval entre los temas de estudio. A excepción de UFAM y UFMT, que ofrecen dos asignaturas, Historia Medieval I e Historia Medieval II, las demás universidades ofrecen un componente - comúnmente llamado Historia Medieval-centrado en estudios medievales. Ese tema está presente en todos los grados, y solo varían los objetivos y la pluralidad de enfoque.

En las siguientes asignaturas, generalmente ofrecidas entre el cuarto y quinto semestre, se identifican los contenidos de estudio centrados en la historia moderna. La oferta de asignaturas en los diseños curriculares que analizan ese período/tema es similar a la configuración presentada para los estudios sobre 
historia medieval. Sin embargo, Unifap, UFMT, UFMA y Unifesspa presentan dos asignaturas centradas en el estudio de ese tema. Las demás universidades tienen en sus diseños curriculares solo una asignatura para estudios relacionados con la llamada historia moderna.

Con respecto al marco temporal, cronológicamente más cercano al nuestro, la historia contemporánea, cualquiera que sea el significado que se le atribuya, parece disfrutar de mayor simpatía entre los responsables de la elaboración de los diseños curriculares analizados. Todos los cursos examinados ofrecen dos materias obligatorias que abordan este contenido. Cuando analizamos únicamente los nombres de las asignaturas que incluyen "historia contemporánea", nos damos cuenta de que UFPA presenta una sola asignatura centrada en ese tema de estudio. Sin embargo, en el diseño curricular de la referida institución, las asignaturas no siguen esta división clásica de nombres (Historia Antigua, Historia Medieval, Historia Moderna e Historia Contemporánea). Hay otras asignaturas con otros nombres que se dedican al estudio de temas relacionados con el marco temporal de la historia contemporánea, tales como "Matrices del pensamiento historiográfico del siglo XIX", "Matrices del pensamiento historiográfico del siglo XX", "Historia de la República de Brasil (1945 - tiempo presente)", "Historia del imperialismo", "Formación de Estados nacionales y regímenes autoritarios en Latinoamérica" e "Historia de las revoluciones"3.

Esa clásica división temática/temporal aparece de manera similar en las asignaturas que estudian la historia de Brasil. En los diseños curriculares analizados prevalece la misma división entre las referidas asignaturas distribuidas a lo largo de los cursos, sin divergencia. Predomina una configuración en la que la historia de Brasil se divide y se presenta en tres bloques temáticos: Colonia, Imperio y República, a excepción de la carrera de Historia de la UFPA. No hay diferencias consistentes entre las materias, pero hay algunos cambios en el título de la asignatura, como muestra el diseño curricular de la UFRR. En esa universidad, las asignaturas se denominan "Historia e historiografía de Brasil" más el número romano I, II, III y IV para distinguirlas. Sin embargo, sus programas de curso muestran que la misma selección temática prevalece en términos de aproximaciones a los períodos colonial, imperial y republicano.

Más que una división por tema y período, percibimos el predominio de una interpretación de la historia como ciencia. La historia política clásica predomina en la configuración que los diseños curriculares presentan en cuanto a la oferta de asignaturas y sus problemas de estudio. Del mismo modo, hay una distribu-

Como el diseño curricular de la UFPA no sigue el "modelo clásico" en la distribución de asignaturas, se utilizó como criterio la mayor cantidad de objetivos presentes en cada programa de curso sobre temas relacionados con el período de la llamada historia contemporánea. Por eso hay tres asignaturas indicadas en la tabla 1 sobre la llamada historia contemporánea. 
ción cronológica del tiempo en la secuenciación de asignaturas a lo largo de la formación. Por lo tanto, los primeros se dirigen al estudio de temas más antiguos, mientras que los últimos temas están dirigidos al estudio de cuestiones relacionadas con la contemporaneidad o el tiempo reciente. Las carreras comienzan con temas centrados en los estudios de la historia antigua, pasan a la historia medieval y la historia moderna y llegan, por fin, a la historia contemporánea.

\section{El lugar de la enseñanza y el libro de texto en las carreras de grados de Historia}

Los análisis que surgen del material estudiado apuntan a una configuración de cómo se entiende, estudia y distribuye el tiempo en los planes curriculares de las carreras de grado aquí analizadas. También demuestra el lugar ocupado por cada asignatura y cada tema estudiado, e identifica el tiempo de estudio obligatorio asignado a los temas respectivos. En ese sentido, podemos mapear, registrar y discutir el lugar que ocupan la enseñanza de la historia y el libro de texto en las asignaturas. Por lo tanto, es posible analizar cuántas y qué asignaturas obligatorias ofrecen los diseños curriculares durante la formación inicial del profesorado de Historia con esas materias de estudio. La tabla 2 muestra los resultados catalogados en los dibujos curriculares analizados.

Tabla 2. Relación institución y asignaturas obligatorias sobre la enseñanza de la historia y los libros de texto

\begin{tabular}{lcccc}
\hline & & \multicolumn{3}{c}{ Asignaturas obligatorias } \\
\cline { 3 - 5 } $\begin{array}{l}\text { Institución (año de } \\
\text { aprobación del PPC) }\end{array}$ & $\begin{array}{c}\text { Carga horaria } \\
\text { total (en horas) }\end{array}$ & $\begin{array}{c}\text { Total de } \\
\text { asignaturas }\end{array}$ & $\begin{array}{c}\text { Asignaturas sobre } \\
\text { enseñanza de la } \\
\text { historia }\end{array}$ & $\begin{array}{c}\text { Asignaturas } \\
\text { sobre libro de } \\
\text { texto }\end{array}$ \\
\hline UFAM (2006) & 2.865 & 41 & 3 & - \\
\hline Unifap (2007) & 3.420 & 44 & 4 & - \\
\hline UFMT (2009) & 3.268 & 39 & 1 & - \\
\hline UFPA (2011) & 3.260 & 45 & 8 & 1 \\
\hline UFT (2011) & 2.805 & 38 & 1 & - \\
\hline UFRR (2012) & 2.810 & 45 & 4 & - \\
\hline UFAC (2013) & 2.930 & 39 & 2 & - \\
\hline UFMA (2014) & 2.865 & 36 & 1 & - \\
\hline UNIR (2014) & 3.560 & 32 & 2 & - \\
\hline Unifesspa (2018) & 3.216 & 45 & 8 & 2 \\
\hline Ufopa (2018) & 3.470 & 37 & 2 & \\
\hline Total & 34.469 & 432 & 36 & \\
\hline
\end{tabular}

Fuente: elaboración propia. 
Los resultados encontrados por los identificadores utilizados muestran que UFPA y Unifesspa aparecen con ocho asignaturas curriculares obligatorias, de un total de cuarenta y cinco en cada diseño curricular, destinadas a discutir temas relacionados con la enseñanza de la historia. Sin embargo, hay otras asignaturas en el diseño curricular de UFPA que también se dedican al análisis del estudio sobre la enseñanza de la historia, aunque no presenten los términos de identificación que se utilizan en la investigación ${ }^{4}$. La Unifap ofrece en su diseño curricular cinco asignaturas, de un total de cuarenta y cuatro, centradas en el debate sobre la enseñanza de la historia, que son cuatro seminarios de práctica docente y una asignatura denominada "Metodología de la enseñanza de la historia". UFRR, por su parte, aparece con cuatro asignaturas tituladas "Prácticas de enseñanza I, II, III y IV". UFAM tiene tres asignaturas obligatorias (de un total de cuarenta y cinco) que son "Metodología de enseñanza de la historia", "Práctica integrada I" y "Práctica integrada II" con sesenta horas de clases cada una. A seguir, UFAC ofrece treinta y nueve asignaturas obligatorias de las cuales dos mencionan la enseñanza de la historia. Esa cantidad de material sobre la enseñanza de la historia es la misma que ofrecen las universidades UNIR y Ufopa. UFT, UFMA y UFMT ofrecen solo una materia que hace mención nominal a la enseñanza de la historia. Sin embargo, vale la pena señalar que el diseño curricular de UFMA presenta una configuración distinta. En diecinueve de sus asignaturas, que tratan de diversos temas, se inserta un objetivo de estudio que relaciona el tema específico de esa asignatura con la educación primaria y secundaria ${ }^{5}$. Esa configuración también la encontramos en el diseño curricular de UFMT, pues en veinte asignaturas — de temas diversos- se encuentra un objetivo de estudio que se dedica a la enseñanza de la historia.

Esa primera muestra ya presenta algunas indicaciones del tiempo asignado para los debates sobre la enseñanza de la historia dentro de los diseños curriculares de las instituciones analizadas. Como es notable, existen diferencias significativas entre el número de asignaturas centradas en este debate. No obstante, en los programas de curso de cada asignatura, la mención acerca del estudio sobre la enseñanza no garantiza que el debate será llevado a cabo. Existen muchas variables entre lo que se prescribe en el texto formal y su aplicabilidad.

Sin embargo, no podemos negar que los PPC son documentos legales y formales de singular importancia, ya que definen un conjunto de prácticas y regulaciones dentro de los cursos en que se aplican. De igual forma, podemos extender la reflexión y afirmar que es posible desarrollar discusiones sobre la

Existen asignaturas como "Práctica curricular supervisada I, II, III y IV" que también analizan cuestiones relacionadas con la enseñanza de la historia.

5 Como ejemplo, la asignatura Historia Antigua define que uno de sus objetivos de estudio es relacionar la "[h] istoria antigua en educación primaria y secundaria" (UFMA, 2014, p. 60). 
enseñanza o el libro de texto, aunque esos temas no aparezcan en la escritura formal de los diseños curriculares. Hay muchas variables en cuestión, como la formación, el interés, la sensibilidad y la autonomía de los docentes en la ejecución de las disciplinas.

Los PPC analizados, sin embargo, señalan sus fundamentos epistemológicos, sus principios legales y sus marcos teóricos. De esa forma, su configuración nos permite comprender sus requisitos, obligaciones y, por extensión, lo que consideran de mayor o menor importancia en el proceso de formación de un profesor de Historia.

Esa primera identificación de los temas curriculares dirigidos al debate sobre la enseñanza, realizada en el PPC, también indica cuánto tiempo se dedica, durante el proceso inicial de formación, a las reflexiones sobre la enseñanza de cada área en forma específica. Sin embargo, el análisis no se trata de la cuestión puramente cuantitativa del tiempo, aunque sean datos importantes, ya que permiten aludir al tiempo asignado para los debates relacionados con la enseñanza de la historia. La reflexión nos permite cuestionar la selección de un determinado tiempo para ciertos contenidos a expensas de otros. El análisis permite percibir la disparidad (en términos de tiempo y cantidad) entre las asignaturas que abordan los "temas clásicos" y las que se dirigen a los estudios sobre la enseñanza de la historia y los libros de texto.

En esa dimensión, no sería improbable decir que cuanto menos tiempo es dedicado a los debates relacionados con la enseñanza, durante la formación inicial, menos condiciones serán construidas para la reflexión y la construcción de cambios en la práctica docente. En otras palabras, cuanto más corto sea el tiempo asignado a las reflexiones sobre la enseñanza y, en consecuencia, a la formación docente de los futuros docentes, menores serán las condiciones para replantear la práctica docente en educación primaria y secundaria a partir del espacio de formación inicial.

La cantidad de material sobre la enseñanza de la historia muestra una disparidad significativa en los diseños curriculares analizados. La situación es aún más desafiante cuando analizamos las asignaturas específicas destinadas a los estudios sobre la principal herramienta de trabajo de los futuros y actuales docentes: el libro de texto.

De los once diseños curriculares, solo dos ofrecen asignaturas obligatorias específicas para analizar el libro de texto. UFPA y Unifesspa tienen una asignatura específica para estudiar/analizar el libro de texto. En UFPA, de acuerdo con el plan del curso, la asignatura "Práctica curricular continua VItexto didáctico: producción y usos" está destinada al "análisis y uso de libros 
de texto de historia, transposición didáctica de textos historiográficos, reflexión sobre procedimientos de evaluación" (UFPA, 2011, p. 61). En Unifesspa, la asignatura "Práctica curricular continua II-historia y enseñanza: texto didáctico, producción y uso" se centra en el

Análisis del proceso de construcción del material didáctico de la historia para la educación primaria y secundaria y sus complejas relaciones con el proceso de formación inicial del profesorado. Se problematizará la relación entre el libro de texto y el mercado editorial, a fin de comprender las posibilidades y los límites en la producción de libros de texto en disputas que involucran al Estado, el mercado y la sociedad. También se promoverán debates y experiencias sobre las posibilidades de uso del libro de texto en la enseñanza de docentes en formación. Desarrollar actividades en el aula para problematizar los desafíos y explorar las posibilidades de utilizar el libro de texto como herramienta de investigación en la vida diaria de los maestros de educación primaria y secundaria. (Unifesspa, 2018, p. 125)

Si ampliamos la búsqueda por asignaturas que incluyen ese tema entre sus objetivos, aunque no sean específicas sobre los libros de texto, surge otra configuración. La tabla 3 muestra los resultados de los datos de los diseños curriculares que incluyen el libro de texto entre los temas para la reflexión.

Tabla 3. Relación institución-materia que incluye el libro de texto

\begin{tabular}{lccc}
\hline \multirow{2}{*}{$\begin{array}{c}\text { Institución } \\
\text { (año de probación del PPC) }\end{array}$} & $\begin{array}{c}\text { Carga horaria } \\
\text { total (en horas) }\end{array}$ & $\begin{array}{c}\text { Asignaturas obligatorias } \\
\text { Total de } \\
\text { asignaturas }\end{array}$ & $\begin{array}{c}\text { Asignaturas que } \\
\text { incluyen el libro de texto }\end{array}$ \\
\hline UFAM (2006) & 2.865 & 41 & 1 \\
\hline Unifap (2007) & 3.420 & 44 & - \\
\hline UFMT (2009) & 3.268 & 39 & - \\
\hline UFPA (2011) & 3.260 & 45 & 1 \\
\hline UFT (2011) & 2.805 & 38 & 1 \\
\hline UFRR (2012) & 2.810 & 45 & 1 \\
\hline UFAC (2013) & 2.930 & 39 & - \\
\hline UFMA (2014) & 2.865 & 36 & 1 \\
\hline UNIR (2014) & 3.560 & 32 & 2 \\
\hline Unifesspa (2018) & 3.216 & 45 & 9 \\
\hline Ufopa (2018) & 3.470 & 37 & 1 \\
\hline Total & 34.469 & 432 & \\
\hline
\end{tabular}

Fuente: elaboración propia. 
La UFAM ofrece la asignatura Práctica Integrada I. Su plan de estudios muestra que se llevarán a cabo reflexiones sobre las diversas narrativas y documentos que pueden ser utilizados como producción histórica y que pueden ser apropiados para el universo de la escuela primaria y secundaria. Entre esos documentos se destacan la fotografía, el periódico, la literatura, el cine, la música, la pintura y otras expresiones artísticas, "además del libro de texto como representación" (UFAM, 2006, p. 38).

El diseño curricular de licenciatura en Historia en la UFAC presenta una configuración similar. En esa institución no hay una asignatura específicamente sobre el libro de texto. Sin embargo, una de sus asignaturas -Enseñanza de la Historia II- está centrada en el debate sobre la enseñanza de la historia y menciona los materiales didácticos como uno de los objetivos a analizar. Según el proyecto pedagógico curricular, esta asignatura

[a]naliza la escuela en sus relaciones con la enseñanza de la historia: las posibles metodologías; las Leyes de Directrices de Bases (LDB), los Parámetros Curriculares Nacionales (PCN) y el currículo de historia; materiales didácticos; la educación de profesores de Historia y su inserción en el mercado laboral; nuevas narrativas en la enseñanza de la historia (cine, fotografía, periódico, música, literatura, etc.); y, una investigación de campo sobre la situación actual de la enseñanza de la historia. (UFAC, 2013, p. 70, énfasis mío)

Tal situación es similar a la encontrada en el diseño curricular del curso de Historia en la UNIR. Como se muestra, el curso consta de treinta y tres asignaturas obligatorias, entre las cuales está Didáctica. De acuerdo con el proyecto pedagógico curricular, la asignatura Didáctica está destinada a

[r]elacionar las opciones teóricas y las decisiones didáctico-pedagógicas en la elaboración de planes estratégicos para el proceso de enseñanza-aprendizaje de la historia y su evaluación, así como proporcionar una reflexión sobre los criterios para la selección y el uso de los libros de texto, permitiendo a nuestro alumno su futuro desempeño como docentes de Historia. (UNIR, 2013, p. 62, énfasis mío)

La UFRR también presenta la misma configuración. El proyecto políticopedagógico del curso ofrece treinta y ocho asignaturas obligatorias. Entre ellas se encuentra la asignatura "Práctica docente IV: recursos didácticos y didácticos", ofrecida en el cuarto semestre. De acuerdo con el proyecto pedagógico curricular, esa asignatura está destinada a promover

[o]rientación para el comienzo de la práctica docente; contacto con la escuela para llevar a cabo la pasantía supervisada; recursos didácticos: TV y proyector multimedia; uso de libros de texto y uso de mapas; documentos escritos y no escritos en el aula. Observación en el instituto. (UFRR, 2012, p. 11, énfasis mío) 
La UFT no es una excepción a la regla. Su diseño curricular presenta una sola asignatura para estudiar todo el universo de temas relacionados con la enseñanza de la historia. Se trata de la asignatura "Metodología de la enseñanza de la historia", la cual presenta, entre sus objetivos de estudio, el análisis "del libro y del material didáctico de la historia" (UFT, 2011, p. 72).

Tres universidades no hacen mención textual formal, en sus diseños curriculares, a cualquier reflexión sobre libros de texto: Unifap, UFMT y UFMA. ¿Qué nos puede decir ese panorama? ¿Qué lecturas y significados ofrecen sobre los diseños curriculares para los cuales están siendo capacitados los futuros docentes de Historia de la educación primaria y secundaria? ¿Qué temas han sido considerados los más importantes y, por lo tanto, obligatorios entre los temas estudiados durante la formación inicial del profesorado?

La existencia de asignaturas que insertan el libro de texto entre sus objetivos de estudio puede llevar a creer que los estudios sobre ese tema (en los currículos) son suficientes. Una lectura rápida puede plantear el argumento de que hay una gran variedad de contenido y temas para estudiar durante el período de formación inicial. Sin embargo, no podemos sacar conclusiones apresuradas.

Cada asignatura en las carreras de pregrado en Brasil tiene una duración promedio de cuatro meses. Las que incluyen el análisis de libros de texto entre sus objetivos de estudio también incluyen un promedio de otros cinco temas/objetivos. Con esta configuración, el tiempo disponible para estudiar cada objetivo propuesto se reduce a menos de un mes. Como regla general, esas materias tienen una clase de cuatro horas a la semana. Debido a la cantidad de objetivos/temas que ofrecen las asignaturas, al final quedan menos de cuatro días de clases para analizar cada uno de los objetivos presentados. Eso significa que, de las once carreras de licenciatura en Historia analizadas en este texto, solo dos (UFPA y Unifesspa) ofrecen una asignatura específicamente enfocada en estudios de libros de texto. La carrera de la Ufopa distribuye estudios de libros de texto y otros objetivos en dos asignaturas. Los cursos en UFAM, UFT, UFRR, UFAC y UNIR insertan las discusiones en una sola asignatura. Finalmente, tres cursos, como ya se ha mostrado (Unifap, UFMT y UFMA), no presentan ninguna discusión en sus temas.

\section{Consideraciones finales}

¿Cuál es la formación que delinean estos diseños curriculares en general y con respecto al análisis y utilización de los libros de texto en particular? ¿Qué contenidos se estudian y cuáles son silenciados al seleccionar y ofrecer los temas para cada asignatura? 
Se ha convertido en una consigna, en nuestro tiempo actual, el hecho de que los libros de texto deben transformarse en un objeto de investigación en educación primaria y secundaria, y que los docentes deben transformarlos y usarlos como objetos de investigación en el aula; que no pueden usarlos como depósitos de verdades absolutas; que necesitan usar el libro de texto como un recurso adicional y no como la única herramienta de trabajo. Esas expresiones se convirtieron en jerga, en ecos vacíos arrojados al viento.

El problema no se resolverá simplemente señalando que los docentes deben replantear sus usos de los libros de texto. ¿Qué condiciones prácticas tienen los docentes para promover tal cambio? ¿Qué asignaturas ofrecen, durante el período de formación inicial, las condiciones para experimentar esa problematización entre los alumnos? ¿Cuánto tiempo se ofrece a los docentes durante la capacitación inicial para revisar el libro de texto?

Los diseños curriculares de los cursos de grado son proyectos construidos a través de la interpretación que sus autores elaboran a lo largo del tiempo. Por extensión, también constituyen una forma de apropiación del pasado, de acción en el presente y de proyección y construcción del futuro.

Como ya se mencionó, los diseños curriculares son documentos muy complejos. Se fabrican en un campo de tensión y disputas permanentes. Deben cumplir con un conjunto de prerrogativas institucionales que regulan y definen las pautas y regulaciones para las carreras de grado. Sin embargo, también son documentos construidos por docentes que trabajan en los respectivos departamentos. Los PPC no son construcciones extrañas. Fueron preparados por docentes que trabajan en la formación de docentes. Cuando los diseños curriculares experimentan cambios, esos son realizados por profesionales que trabajan en las respectivas universidades. Por lo tanto, esos docentes -en el conjunto de las disputas poder - son responsables de la inserción o del mantenimiento de cada asignatura dentro de los diseños curriculares. Ellos (es decir, nosotros) son responsables de garantizar la ausencia de asignaturas destinadas a reflexiones y aprendizaje sobre la enseñanza y los libros de texto.

La configuración de los diseños curriculares analizados muestra que existe una gran asimetría entre las asignaturas obligatorias destinadas a los estudios de los "temas clásicos" (que siguen el modelo cuatripartito) y las asignaturas obligatorias centradas en las reflexiones sobre la enseñanza. La asimetría es aún mayor cuando la comparamos con las asignaturas dirigidas a estudios sobre el libro de texto. En mayor o menor medida, esos diseños curriculares contienen las marcas de los docentes que trabajan en los respectivos departamentos. 
Es importante registrar que la construcción de los proyectos políticos curriculares es responsabilidad de los docentes que actúan en los departamentos donde se ubican los cursos de formación del profesorado. Son esos profesionales quienes determinan la selección de asignaturas, temas y objetivos de estudio. Los PPC cuentan, en gran medida, con el acuerdo de sus docentes en mantener determinado modelo de propuesta curricular que, con algunas excepciones, permanece anclado en la división clásica de la historia en cuatro bloques temáticos principales. ¿Basados en qué motivos es posible argumentar que una asignatura obligatoria sobre historia antigua, o cualquier otra historia, es más o menos importante que una asignatura destinada a analizar el libro de texto de historia? No se debe olvidar que el libro de texto sigue siendo el principal —a veces la única- herramienta de trabajo del docente en la educación primaria y secundaria en Brasil. Sin embargo, aunque no se verbalice que se percibe que un tema es más importante que el otro, eso se manifiesta a través del mantenimiento de algunas asignaturas temáticas.

Los diseños curriculares analizados, por lo tanto, establecen, delimitan y definen un tiempo determinado para cada tema que consideran importante en el proceso de formación inicial del docente. Además, excluyen e inviabilizan otras discusiones. El tiempo destinado a cada asignatura o contenido es bastante significativo y demuestra los valores, intereses y prioridades de los docentes que trabajan en cada una de las facultades. La ausencia, por lo tanto, de un mayor debate sobre la enseñanza de la historia y del libro de texto también revela cómo los formadores de docentes y la propia ciencia histórica (como área de conocimiento) entienden la formación docente en la licenciatura en Historia.

Desde este ángulo de percepción, antes de que se defiendan determinados discursos (sobre la manutención de una u otra asignatura), sería importante dirigir la atención en las relaciones de poder que configuran los cursos de grado donde san formados el profesorado. Estas relaciones de poder pueden contribuir a crear estrategias que posibiliten el análisis de los libros de texto como objeto de investigación durante el período inicial de formación docente. Será ilusión creer que, después de graduarse, trabajar en un aula por largas horas y en condiciones no siempre favorables, los maestros transformen - a través de la "intervención divina"- el libro de texto en un instrumento de investigación, en una rutina escolar.

\section{Agradecimientos}

Me gustaría agradecer a los miembros (estudiantes e investigadores) del laboratorio y grupo de investigación Interpretación del tiempo: enseñanza, memoria, narrativa y política (iTemnpo) a través de lecturas, críticas y sugerencias. 


\section{Referencias}

Abud, K. (1998). Currículos de história e políticas públicas. Os programas de história do Brasil na escola secundária. En C. M. F. Bittencourt (org.), O saber histórico em sala de aula (pp. 28-41). Contexto.

Arroyo, M. G. (2013). Currículo, território em disputa. Vozes.

Bittencourt, C. M. F. (2009). Ensino de História: fundamentos e métodos. Cortez.

Cassiano, C. C. F. (2017). Política e economia de mercado do livro didático no século XXI: globalização, tecnologia e capitalismo na educação básica nacional. En H. Rocha, L. Reznik y M. de Souza (orgs.), Livros didáticos de história: entre políticas e narrativas (pp. 83-100). FGV.

Cavalcanti, E. (2016). Livro didático: produção, possibilidades e desafios para o ensino de História. Revista História Hoje, 5(9), 262-284. https://doi.org/10.20949/rhhj.v5i9.219

Cavalcanti, E. (2018a). A história encastelada e o ensino encurralado: reflexões sobre a formação docente dos professores de história. Educar em Revista, 34(72), 249-267. https://revistas.ufpr.br/ educar/article/view/60111

Cavalcanti, E. (2018b). História, livro didático e formação docente: produção, limites e possibilidades. Antíteses, 11(22), 516-532. http://dx.doi.org/10.5433/1984-3356.2018v11n22p516

Choppin, A. (2004). História dos livros e das edições didáticas: sobre o estado da arte. Educação e Pesquisa, 30(3), 549-566. https://doi.org/10.1590/S1517-97022004000300012

González, M. P. (2004-2005). Los profesores y la transmisión de la historia argentina reciente: entre el currículum y el contexto. Trabajos y Comunicaciones, (30-31). https://www.trabajosycomunicaciones.fahce.unlp.edu.ar/article/view/TYC2004n30-31a03

Guimarães, S. y Couto, R. C. (2008). A formação de professores de história no Brasil: perspectivas desafiadoras de nosso tempo. En E. Zamboni y S. Guimarães (orgs.), Espaços de formação do professor de história (pp. 101-130). Papirus.

Instituto Brasileiro de Geografia e Estatística, IBGE (2020). ZEE Amazônia Legal. https://www.ibge.gov. $\mathrm{br} /$ geociencias/cartas-e-mapas/mapas-regionais/15844-zee-amazonia-legal.html? $=\& \mathrm{t}=0$-que-e

Laville, C. (1999). A guerra das narrativas: debates e ilusões em torno do ensino de História. Revista Brasileira de História, 19(38), 125-138. http://dx.doi.org/10.1590/S0102-01881999000200006

Mathias, C. L. K. (2011). O ensino de história no Brasil: contextualização e abordagem historiográfica. História Unisinos, 15(1), 40-49. http://revistas.unisinos.br/index.php/historia/article/view/959

Melo, M. C. D. (2015). A formação de professores de História em Portugal: práticas pedagógicas e investigativas. Revista História Hoje, 4(7), 41-61. https://doi.org/10.20949/rhhj.v4i7.177

Miranda, S. R. y Luca, T. R. (2004). O livro didático de história hoje: um panorama a partir do PNLD. Revista Brasileira de História, 24(48), 123-144. http://dx.doi.org/10.1590/S0102-01882004000200006

Ministerio de Educación. (2019, 20 de diciembre). Resolución 2 de 2019. Por medio de la cual se reglamenta parcialmente los cursos de grado para la formación del profesorado en Brasil. http://portal.mec.gov. br/index.php?option=com_docman\&view $=$ download\&alias $=135951-\mathrm{rcp} 002-19 \& I t e m i d=30192$

Monteiro, A. M. (2007). Professores de história: entre Saberes saberes e práticas. Mauad X.

Monteiro, A. M. (2013). Formação de professores: entre demandas e projetos. Revista História Hoje, 2(3), 19-42. https://doi.org/10.20949/rhhj.v2i3.63

Moreira, A. F. B. y Silva, T. T. (2000). Currículo, cultura e sociedade. Cortez Editora. 
Munakata, K. (2012). O livro didático: alguns temas de pesquisa. Revista Brasileira de História da Educação, 12(3), 179-197. http://www.periodicos.uem.br/ojs/index.php/rbhe/article/view/38817

Oliveira, M. D., Cainelli, M. R. y Oliveira, A. F. B. (2008). Ensino de História: múltiplos ensinos em múltiplos espaços. EDUFRN.

Pagès, J. (2004). Enseñar a enseñar Historia: la formación didáctica de los futuros profesores. En J. A. Gómez y M. E. Nicolás (coords.), Miradas a la Historia: reflexiones historiográficas en recuerdo de Miguel Rodríguez Llopis (pp. 155-178). Universidad de Murcia, Servicio de Publicaciones.

Rocha, H. (2019). Desafios presentes nos livros didáticos de História: narrar o que ainda está acontecendo. Revista História Hoje, 7(14), 86-106. https://doi.org/10.20949/rhhj.v7i14.466

Rocha, H., Reznik, L. y De Souza, M. (orgs.) (2017). Livros didáticos de história: entre políticas e narrativas. FGV.

Rodríguez, M. V. (2008). Políticas de formação de professores: as experiências de formação inicial em Argentina, Chile e Uruguai. Nuances: estudos sobre Educação, 15(16), 119-139. https://doi. org/10.14572/nuances.v15i16.180

Silva, C. B. y Rossato, L. (2013). A didática da história e o desafio de ensinar e aprender na formação docente inicial. Revista História Hoje, 2(3), 65-85. https://doi.org/10.20949/rhhj.v2i3.65

Tardif, M. (2014). O trabalho docente: elementos para uma teoria da docência como profissão de interações humanas. Vozes.

Universidade Federal de Mato Grosso, UFMT (2009). Projeto Político Pedagógico do Curso de Licenciatura em História. http://sistemas.ufmt.br/ufmt.ppc/PlanoPedagogico/Download/131

Universidade Federal de Roraima, UFRR (2012). Projeto Político Pedagógico do Curso de Licenciatura em História. https://cutt.ly/3fd6uZk

Universidade Federal do Acre, UFAC (2013). Projeto Político Pedagógico do Curso de Licenciatura em História. Recuperado de envío por correo personal de una investigadora de la institución.

Universidade Federal do Amapá, Unifap (2007). Projeto Político Pedagógico do Curso de Licenciatura em História. http://www2.unifap.br/historia/files/2013/04/licenciaturahistoria.pdf

Universidade Federal do Amazonas, UFAM (2006). Curso de Licenciatura Plena em História Projeto Pedagógico. https://cutt.ly/Wfd6sXO

Universidade Federal do Maranhão, UFMA (2014). Projeto Político Pedagógico do Curso de Licenciatura em História. https://sigaa.ufma.br/sigaa/public/curso/ppp_curso.jsf?lc $=$ pt_BR\&lc $=$ pt_BR\&id $=85811$

Universidade Federal do Oeste Do Pará, Ufopa (2018). Projeto Político Pedagógico do Curso de Licenciatura em História. http://www2.ufopa.edu.br/ufopa/arquivo/proen-cursos-portarias-ppcs/ppc-licenciatura-historia

Universidade Federal do Pará, UFPA (2011). Projeto Político Pedagógico do Curso de Licenciatura em História. Recuperado de envío por correo personal de una investigadora de la institución.

Universidade Federal do Sul e Sudeste Do Pará, Unifesspa (2018). Projeto Político Pedagógico do Curso de Licenciatura em História. Unifesspa. https://historia-maraba.unifesspa.edu.br/images/arquivos/ PPC-HISTORIA-2018-ATUALIZADO.pdf

Universidade Federal do Tocantins, UFT (2011). Projeto Político Pedagógico do Curso de Licenciatura em História. http://download.uft.edu.br/?d=14edae36-efb0-4382-9da7-d8267c9e5cd9 